\title{
Disciplines non linguistiques en Langues Vivantes Étrangères : quelle prise en compte des effets de contextes pour améliorer les enseignements ?
}

\author{
Christian MICHAUD \\ Laboratoire CRIS EA 647, Université Claude Bernard Lyon 1
}

\section{Résumé}

L'objet de cet article est de présenter, de comprendre les pratiques d'enseignement de professeurs qui ont en charge une classe de discipline dite non linguistique (DNL) en langue vivante étrangère (LVE) en sections européennes. Cette recherche est consécutive à la mise en place de la formation préparant à la certification DNL en LVE à l'IUFM sur l'Académie de Lyon (2006-2010). L'enseignement de la DNL dans la LVE utilise le contexte de la langue étrangère pour transmettre les contenus de la DNL et s'appuie sur le contexte spécifique de la DNL pour faire progresser les élèves à la fois dans la discipline et la langue. Cela constitue la spécificité de cet enseignement (Coste, 2003; Duverger, 2007; Causa, 2009). Cet enseignement pose des questions au niveau des contenus scientifiques à transmettre, des documents utilisés et de la contextualisation didactique. Ainsi, les conceptions des acteurs de la DNL sont en lien direct avec les contextes et les effets que ceux-ci produisent. Comment prendre en compte ces effets de contextes pour améliorer l'efficacité des enseignements de la DNL? Sur le plan méthodologique, nous avons recueilli deux types de données : les questionnaires adressés à l'ensemble des acteurs concernés par la DNL (formateurs et stagiaires) et les artéfacts relatifs aux enseignements de la DNL dans les portfolios des stagiaires de l'IUFM de Lyon 1. L'analyse des questionnaires et des artéfacts sur les conceptions des enseignants est réalisée à partir du modèle théorique Knowledge-Value-Practice (Clément, 2006). A travers les conceptions des acteurs, nous repérons les effets de contextes, nous analysons comment ceux-ci sont utilisés et proposons une première ébauche qualitative d'une contextualisation didactique propre à l'enseignement de la DNL en LVE sur la base des disciplines rencontrées.

\section{Mots-clés}

Contextes, discipline non linguistique, langue étrangère, portfolio.

\begin{abstract}
This work presents the methods used in teaching a nonlinguistic disciple (NLD) in a foreign language (FL) in European sections. This research is conducted in relation with the training of teachers preparing an exam for NLD at the IUFM Lyon 1 between 2006- 2010. The teaching of a NLD uses the context of the FL to transmit subject content and depends on its own context to develop both knowledge and skills of pupils in the NLD and the FL. This is the specificity of this type of teaching (Coste, 2003 ; Duverger, 2007 ; Causa, 2009). This teaching has raised many questions about the scientific contents to be transmitted, the documents to be used, and the didactic process/ context involved in the NLD and FL. The conceptions of actors teaching NLD in FL are in direct relation to the contexts and the effects produced. How can the teachers take into account the effects of contexts to improve their practice? Regarding methodology, this research was conducted with two groups: the experts who were in charge of teacher training in NLD in a FL and the teacher trainees who developed artifacts related to NLD in a FL in their portfolios at IUFM. Experts and trainee teachers were asked to fill in a paper questionnaire. The results were analyzed on the basis of the Knowledge-Practice-Value model (Clement, 2006). The paper questionnaire and portfolios help us to understand strategies used to teach NLD in FL. The results allow us to draw up the basis of a didactic contextualization of NLD in FL.
\end{abstract}

\section{Keywords}

Contexts, non linguistic disciple, foreign language, portfolio. 


\section{Introduction}

L'objet de cet article est de présenter, de comprendre les pratiques d'enseignement de professeurs qui ont en charge une classe de Discipline dite Non Linguistique (désormais DNL) en Langue Vivante Etrangère (désormais LVE) et en section européenne. Créées en 1992, les sections européennes ont pour objectif de répondre au besoin d'ouverture du collège et du lycée sur le monde. Elles proposent un cursus au collège (classe de $4^{\text {ème }}$ et $3^{\text {ème }}$ ) avec deux heures supplémentaires en langue étrangère mettant l'accent sur la culture et l'histoire de l'Europe. Pour répondre au développement croissant de ces sections européennes sur l'Académie de Lyon et à la demande du Rectorat, l'Institut Universitaire de Formation des Maîtres (désormais IUFM) de Lyon 1 a mis en place une formation DNL en LVE de 2006 à 2010. C'est dans ce cadre que nous avons pu mener cette étude en interrogeant les différents acteurs: d'une part, les formateurs en DNL en vue de comprendre les conceptions développées, et d'autre part, les stagiaires IUFM préparant la certification DNL en vue de comprendre les obstacles rencontrés.

L'enseignement de la DNL dans la LVE utilise le contexte de la langue étrangère comme support de transmission et s'appuie sur le contexte spécifique de la DNL pour faire progresser les élèves à la fois dans la discipline et la langue (compétences linguistiques, sociolinguistiques et pragmatiques). Cela constitue la spécificité de cet enseignement (Coste, 2003 ; Duverger, 2007 ; Causa, 2007, 2009)

L'enseignement d'une DNL en LVE pose cependant des questions au niveau des contenus scientifiques à transmettre, des documents utilisés, de la contextualisation didactique mise en œuvre dans la discipline et la langue. Aussi, les enseignants DNL en LVE sont confrontés à des effets de contextes liés à l'enseignement disciplinaire (DNL) avec les contenus de programmes et de culture scolaire à transmettre (Forquin, 1989), à des situations didactiques, à la pratique de la langue étrangère (Duverger, 2004, 2005), aux valeurs socioculturelles mises en jeu dans la DNL en relation avec l'histoire, la géographie, l'économie, les techniques, etc. des pays où sont parlées la langue première (désormais L1) et la LVE. Ainsi, les conceptions des acteurs de la DNL sont en lien direct avec les contextes et les effets que ceux-ci produisent pour enseigner. Comment prendre en compte ces effets de contextes pour améliorer l'efficacité des enseignements de la DNL ? Comment les professeurs stagiaires, qui préparent la certification DNL, peuvent-ils prendre en compte ces effets de contextes pour surmonter les obstacles?

Ce travail comporte plusieurs parties. Tout d'abord, nous présentons le cadre de la formation DNL en LVE proposée à l'IUFM de Lyon 1 entre 2006 et 2010 ainsi que le portfolio des stagiaires où nous trouvons les traces des expériences de classes DNL en LVE. Nous explicitons ensuite notre approche théorique de l'enseignement DNL dans la LVE. Sur le plan méthodologique, le corpus est constitué de questionnaires et d'extraits de portfolios que nous analysons à partir du modèle Knowledge-Value-Practice (Clément, 2006). À travers les conceptions des acteurs, nous repérons les effets de contextes, nous analysons comment ceuxci sont utilisés et proposons une première ébauche qualitative d'une contextualisation didactique propre à l'enseignement de la DNL en LVE sur la base des disciplines rencontrées. 


\section{Formation DNL en LVE à l'IUFM de Lyon 1}

Depuis 2004, une certification complémentaire ${ }^{1}$ peut être accordée aux enseignants de disciplines non linguistiques pour enseigner leur discipline dans une langue autre que le français, dans le cadre des sections européennes. L'IUFM de l'Académie de Lyon a mis en place cette formation pour les professeurs stagiaires dès 2006. Cette formation comprend trois axes en lien avec le déroulement de l'année de stage en alternance :

- Le premier axe est articulé d'une part sur la connaissance du système éducatif pour ce qui concerne les sections européennes, leurs caractéristiques, les diplômes délivrés, les programmes européens et d'autre part sur les compétences pédagogiques, interculturelles et transdisciplinaires (concevoir et mettre en œuvre une séquence d'apprentissage pour un(e) enseignant(e) chargé(e) de l'enseignement de sa discipline dans une langue étrangère ; travail autour d'une séquence d'apprentissage proposée en section européenne avec des manuels du pays de la langue étrangère ; concevoir un projet d'échange dans une perspective interculturelle et pluridisciplinaire).

- Le second axe concerne la maîtrise de la langue étrangère (LE) pour faire la classe et transmettre les notions et concepts de la discipline enseignée. Les stagiaires peuvent faire une partie du stage de pratique accompagnée dans une section européenne.

- Le troisième axe correspond à l'aisance dans la pratique de la LE. Les stagiaires sont invités à faire un stage professionnel à l'étranger.

La formation spécifique à la DNL, proposée par l'IUFM, répond aux deux premiers axes. Elle est précisée dans le Tableau 1.

\begin{tabular}{|l|c|}
\hline \multicolumn{1}{|c|}{ Formation DNL (2006-2010) } & Durée \\
\hline $\begin{array}{l}\text { Réunion de présentation aux stagiaires de l'IUFM de la DNL en LVE } \\
\text { Présentation de dossiers des années précédentes pour l'inscription }\end{array}$ & $2 \mathrm{~h}$ \\
\hline $\begin{array}{l}\text { Présentation de l'examen par l'IPR }{ }^{2} \text { et le DARIC } \\
\text { Citer les textes, les lire, présentation des programmes européens }\end{array}$ & $3 \mathrm{~h}$ \\
\hline Les systèmes éducatifs européens & $3 \mathrm{~h}$ \\
\hline Que signifie enseigner dans une langue étrangère? & $3 \mathrm{~h}$ \\
\hline Pistes pédagogiques pour la DNL & $3 \mathrm{~h}$ \\
\hline Construction d'une séquence : Mathématiques en anglais & $3 \mathrm{~h}$ \\
\hline Construction d'une séquence : Histoire géographie en allemand & $3 \mathrm{~h}$ \\
\hline Construction d'une séquence : Histoire géographie en espagnol & $3 \mathrm{~h}$ \\
\hline
\end{tabular}

\section{Tableau 1 : Contenu de la formation DNL}

\footnotetext{
${ }^{1} \mathrm{BO} \mathrm{n}^{\circ} 7 \mathrm{du} 12$ février 2004 : une certification complémentaire peut être accordée depuis février 2005 aux enseignants de Disciplines Non Linguistiques (DNL) pour enseigner leur discipline dans une langue autre que le français, dans le cadre des sections européennes.

2 IPR : Inspecteur Pédagogique Régional.

${ }^{3}$ DARIC : Délégué Académique aux Relations Internationales et la Coopération.
} 
Cette formation préparant à la certification DNL est complétée par les stagiaires par des expériences en classes où ils ont l'opportunité d'enseigner la DNL en LVE. Ils relatent ces expériences dans leur portfolio de compétences professionnelles.

\section{Le portfolio révélateur des pratiques DNL en LVE des stagiaires IUFM}

Il existe de nombreuses définitions relatives à l'usage du portfolio : le dossier d'apprentissage, le dossier d'évaluation et le dossier de présentation (Essautier-Bavay, 2004). Le portfolio est :

"le recueil continu, réfléchi et organisé d'une variété de produits authentiques qui documentent le progrès d'un étudiant ou d'un professionnel, ses buts, ses efforts, ses attitudes, ses pratiques pédagogiques, ses accomplissements, ses talents, ses intérêts et son développement au fil du temps »

(Winsor et Ellefson, $1995: 3$ ).

Il s'agit d'une collection structurée, sélective et réflexive des meilleurs travaux (Wolf, 1991 ; Goupil, 1998) pour montrer l'acquisition de compétences. Le portfolio à l'IUFM de Lyon 1 (Michaud et Alin, 2008, 2010 ; Michaud, 2010) est avant tout un portfolio de développement professionnel à visée de compétences (les dix compétences du cahier des charges des maîtres). Il a été mis en place dans le cadre d'une recherche-action-formation participative conduite par le professeur Christian Alin en 2008 et intitulé : "Le portfolio - construction et aide à la construction de compétences professionnelles et d'identité professionnelle ». Le portfolio est utilisé dans la formation à l'IUFM comme outil de certification où les stagiaires doivent apporter la preuve de l'acquisition de compétences. Il se distingue du portfolio européen des langues ${ }^{4}$ qui, lui, est un outil d'information sur le niveau de la langue et des expériences interculturelles, basé sur un cadre européen commun de référence pour les langues.

Nous nous intéressons dans cette recherche aux textes du portfolio, sur la période 2007-2008, qui témoignent de l'appropriation de l'enseignement de la DNL en LVE par les stagiaires. Ces textes, réalisés dans un cadre certificatif, font état des conceptions des stagiaires, des valeurs développées, des pratiques mises en œuvre et des difficultés rencontrées dans le contexte d'enseignement de la DNL dans la LVE.

\section{Approches théoriques : apprentissage, langues et disciplines}

Au niveau de l'apprentissage, les didactiques des disciplines linguistiques et non linguistiques se réfèrent au modèle constructiviste. Jean Piaget prône l'activité physique ou cognitive comme nécessaire au développement de la connaissance. "On ne connaît, en effet, un objet qu'en agissant sur lui et en le transformant» (Piaget, 1970: 85). Vygotski (1934) élargit cette théorie de la connaissance en introduisant l'importance du développement de l'individu dans les phénomènes de communication avec autrui et parle de socio constructivisme. L'apprentissage implique un passage de l'inter psychique entre un maitre et un apprenant à l'intrapsychique par des processus d'intériorisation des notions et concepts chez l'élève. Le processus est médiatisé par le langage. Le langage outil se transforme en langage signe. Ces phénomènes intérieurs, lors de l'apprentissage de concepts nouveaux, se produisent en général tout d'abord dans la L1 qui est celle de nos représentations initiales. Cet apprentissage se

\footnotetext{
${ }^{4}$ Les Portfolios européens des langues sont des applications pédagogiques du Cadre Européen Commun de Référence à différents degrés (primaire, secondaire, adultes) et pour divers publics (élèves, enseignants, familles). Ils constituent des outils encourageant une acquisition positive de la langue et permettent un suivi personnalisé par une évaluation et une auto-évaluation.
} 
réalise dans la zone proximale de développement (Vygotski, 1934) de la langue et de la discipline. La langue étrangère est l'outil, le moyen d'enseignement et d'apprentissage de la DNL « tout en améliorant les compétences en LE (Causa, 2009 : 180).

$\mathrm{Au}$ niveau de la langue, l'enseignement en DNL a pour objectif en particulier de faire progresser les élèves dans le domaine de la communication. La compétence communicationnelle telle que définie dans les compétences à acquérir par les professeurs ${ }^{5}$ pour l'exercice du métier a :

« le souci d'amener les élèves à maîtriser la langue; conduit le professeur à intégrer dans les différentes situations professionnelles l'objectif de maîtrise de la langue orale et écrite par les élèves ; à veiller dans toutes les situations d'enseignement ou éducatives au niveau de langue des élèves, à l'écrit et à l'oral ».

Cette prescription institutionnelle s'appuie sur les compétences suivantes :

- la compétence linguistique qui renvoie aux savoirs et savoir-faire relatifs au lexique, à la syntaxe (connaissances), au phonologique ;

- la compétence sociolinguistique qui recouvre les paramètres socioculturels de l'utilisation de la langue (attitudes dans les différentes situations, registre de langue), valeurs (politesse) ;

- la compétence pragmatique qui fait référence à l'utilisation fonctionnelle des ressources de la langue dans les actes de parole (capacités de raisonnement, pratique).

La compétence communicationnelle se développe dans la LE mais aussi dans la L1 dans le cadre de l'alternance des langues pour enseigner efficacement la DNL (Causa, 2007, 2009; Duverger, 2007, 2009). Cela constitue des effets de contextes sociolinguistiques et langagiers au cours d'interactions didactiques (Anciaux, 2010). Par ailleurs, le Cadre Européen Commun de Référence pour les Langues (CECRL) dépasse le niveau communicationnel et propose une approche actionnelle pour enseigner la langue :

«La perspective privilégiée ici est, très généralement aussi, de type actionnel en ce qu'elle considère avant tout l'usager et l'apprenant d'une langue comme des acteurs sociaux ayant à accomplir des tâches (qui ne sont pas seulement langagières) dans des circonstances et un environnement donnés, à l'intérieur d'un domaine d'action particulier » (p. 15).

Cette approche prône l'entrée par une situation, une tâche, une action sociale dans un contexte qui donne sens à l'activité pour apprendre la langue. L'élève parvient à accomplir une « tâche finale », après un temps d'apprentissage (avec apport et appropriation de connaissances) et d'entrainement. Les temps de travail collaboratif sont souvent privilégiés.

Au niveau de la discipline, le constructivisme prône un élève acteur de ses apprentissages en lui donnant une contrôlabilité sur l'action, un sentiment de performance vers une motivation qui donne sens à l'apprentissage en le rendant désirable "Sont "désirables" des situations qui présentent de la nouveauté plutôt que de l'habitude, donnent l'occasion de faire des choix, conduisent à des questions plutôt qu'à des réponses ; des situations où l'individu se sent largement autonome » (Giordan, 2005 : 58). Les conditions de cette autonomie ne reposent pas seulement sur la mise en action du sujet mais dépendent aussi de l'âge de l'apprenant, de son inhibition dans la phase d'adolescence, de la mise en confiance du groupe par

\footnotetext{
${ }^{5}$ Bulletin officiel $n^{\circ} 29$ du 22 juillet 2010.
} 
l'enseignant et des effectifs toujours plus importants en classe de langue pour pouvoir communiquer efficacement. Cela pose effectivement la question des conditions et du moment propice pour l'introduction de la DNL dans l'enseignement secondaire pour le rendre efficace.

Les disciplines se sont largement inspirées du courant constructiviste sur lequel se sont construites les différentes contextualisations didactiques : en mathématiques sur la situation problème, en sciences physiques et sciences et vie de la terre sur la démarche expérimentale, en sciences et techniques sur l'alternance de méthodes inductives et déductives. Pour conclure ce paragraphe, les interactions langagières en langue étrangère sont à la source de situations socioconstructivistes et de développement cognitif dans l'apprentissage de la DNL et le développement de compétences en LE. La pratique didactisée de l'alternance entre la LE et la L1 est bénéfique à la transmission de la DNL (Causa, 2002) et produit des effets de contextes. La DNL contextualise l'enseignement de la langue étrangère qui trouve un terrain motivationnel à son expression écrite et orale.

\section{Un modèle d'analyse des conceptions des professeurs au niveau des connaissances, valeurs et pratiques : le modèle KVP.}

Les discours rencontrés dans les extraits de portfolio et ou dans les questionnaires font référence aux conceptions des acteurs que nous déclinons selon trois pôles: le pôle des connaissances, le pôle des valeurs et le pôle des pratiques du métier. Ainsi, nous avons privilégié une approche méthodologique de type qualitative qui associe les résultats à ces trois pôles dans le modèle KVP (Knowledge, Value, Practice), (Clément, 2006). Ce modèle (cf. Figure 1) est utilisé à l'origine pour comparer les conceptions de l'enseignement de la biologie dans différents pays dans le cadre du «Projet européen BIOHEAD-Citizen (Biology, Health and Environmental Education for Better Citizenship) »

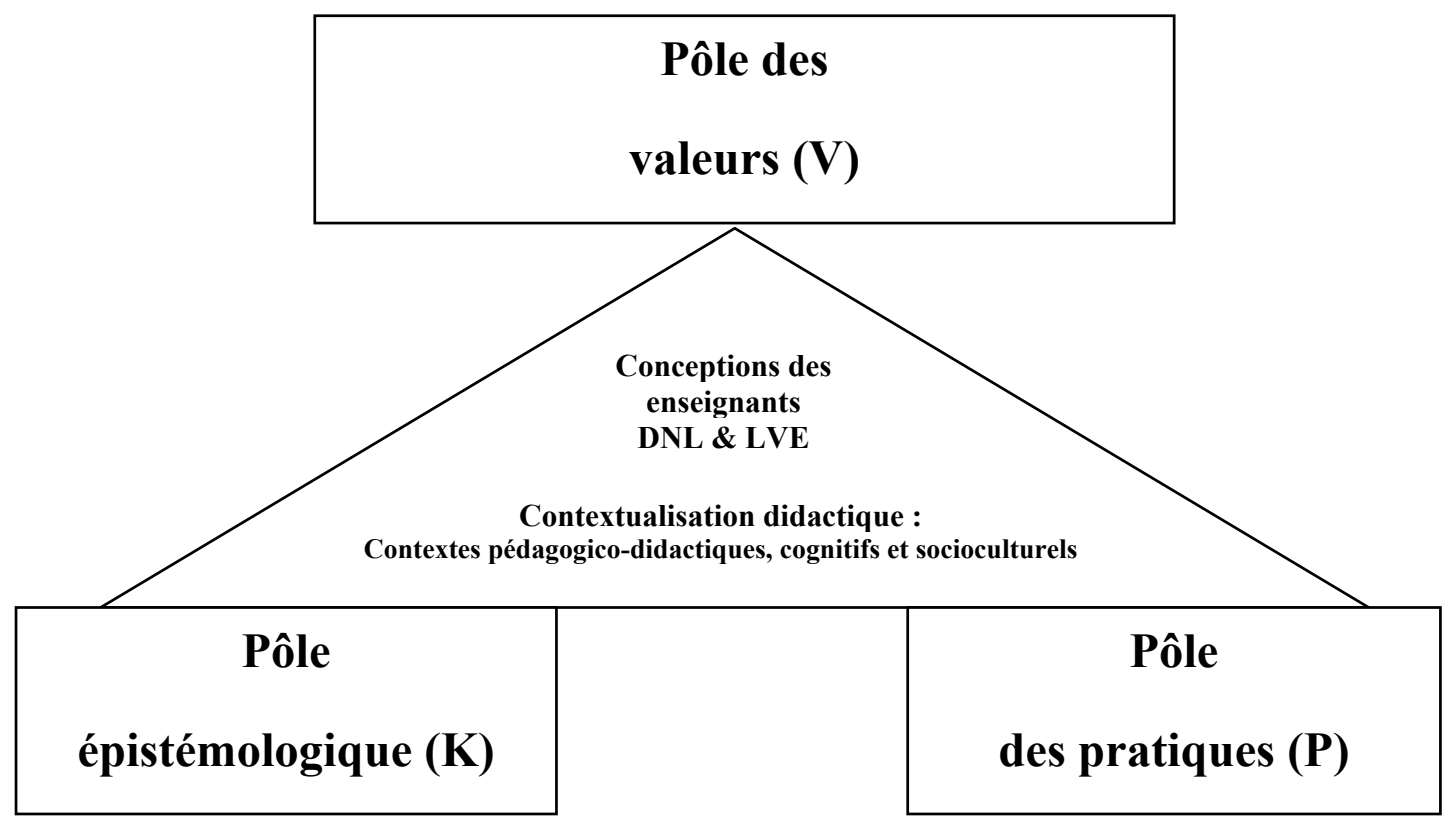

Figure 1 : Modèle KVP

\footnotetext{
${ }^{6}$ Le travail a été développé dans le cadre du projet européen BIOHEAD-Citizen, "Biology,Health and Environmental Education for Better Citizenship ». Vingt équipes de recherche de19 pays ont été impliquées dans ce projet (2004-2008). Les 19 pays ont été choisis pour permettre des études comparatives dans des contextes géographiques, sociaux, économiques, historiques différents : dans ou hors de l'Europe, au nord ou au sud, à l'est ou à l'ouest.
} 
Dans ce modèle, les conceptions de l'enseignant sur la DNL dans la LVE sont en interaction entre les connaissances scientifiques, les systèmes de valeurs personnelles, les pratiques d'enseignement et les contextes didactiques, pédagogiques, cognitifs et socioculturels. Nous explicitons ci-après les trois pôles de ce modèle :

- Pôle des valeurs : Dans le modèle KVP, les valeurs (V) sont considérées dans un sens large comme relevant des coutumes, croyances, idéologies, faits socio et/ou inter culturelles des contextes mais aussi des normes communes et des prescriptions locales.

- Pôle épistémologique ou connaissances : Les connaissances scientifiques (K) sont celles mises en jeu et développées dans le cadre de la contextualisation didactique de la DNL (notions, concepts, méthodes) et de la LE (lexique, syntaxe).

- Pôle des pratiques: Les pratiques sociales (P) auxquelles nous nous référons sont celles que le professeur conduit dans sa classe. Contrairement au pôle épistémologique qui s'appuie sur les disciplines, le pôle des pratiques aborde davantage le coté de la pratique professionnelle contextualisée orientée vers l'action et sa transformation (Rabardel, 2005) : conception des apprentissages, gestion de la classe, différenciation pédagogique, évaluation des apprentissages.

\section{Méthodologie}

Sur le plan méthodologique, nous avons travaillé avec différentes disciplines (mathématiques, physique, histoire géographie, génie civil, communication action bureautique) et à partir de deux types de données :

- un questionnaire (cf. Annexe 1) à destination des formateurs $\mathrm{DNL}^{7}(\mathrm{n}=7)$ et des stagiaires en formation $(n=7)$ à l'IUFM dans la période 2007-2008;

- les extraits de portfolio des stagiaires $(n=7)$ en formation à l'IUFM 2007-2008.

Le questionnaire, transmis par mail aux deux groupes en 2011, interroge tous les formateurs DNL en charge de la formation et les stagiaires sur quatre registres : la pratique de la langue, les manuels et documents pédagogiques utilisés, les contenus d'apprentissage. Au niveau des résultats, la codification des formateurs DNL est donnée dans le Tableau 2 et celle des stagiaires dans le Tableau 3.

\begin{tabular}{|l|c|c|c|c|c|c|c|}
\hline $\begin{array}{c}\text { Code } \\
\text { formateurs }\end{array}$ & BS & LP & VD & JLJ & FP & RC & PG \\
\hline $\begin{array}{l}\text { DNL ou } \\
\text { Statut }\end{array}$ & $\begin{array}{c}\text { Prof. } \\
\text { DNL } \\
\text { Génie } \\
\text { civil }\end{array}$ & $\begin{array}{c}\text { Prof. DNL } \\
\text { Mathématiques }\end{array}$ & $\begin{array}{c}\text { Prof. DNL } \\
\text { Histoire } \\
\text { géographie }\end{array}$ & $\begin{array}{c}\text { Prof. } \\
\text { DNL } \\
\text { Physique } \\
\text { chimie }\end{array}$ & $\begin{array}{c}\text { IPR } \\
\text { Alleman } \\
\text { d } \\
\text { Rectorat }\end{array}$ & $\begin{array}{c}\text { Prof. } \\
\text { Alleman } \\
\text { d } \\
\text { IUFM }\end{array}$ & $\begin{array}{c}\text { Prof. } \\
\text { Angla } \\
\text { is } \\
\text { IUFM }\end{array}$ \\
\hline LVE & Anglais & Anglais & Allemand & Anglais & $\begin{array}{c}\text { Alleman } \\
\text { d }\end{array}$ & $\begin{array}{c}\text { Alleman } \\
\text { d }\end{array}$ & $\begin{array}{c}\text { Angla } \\
\text { is }\end{array}$ \\
\hline
\end{tabular}

Tableau 2 : codification et statut des formateurs DNL

\footnotetext{
${ }^{7}$ Les formateurs DNL sont définis ci-après : enseignants en langue étrangère à l'IUFM, IPR, et enseignants experts ayant une classe de DNL en LVE en section européenne.
} 


\begin{tabular}{|l|c|c|c|c|c|c|c|}
\hline $\begin{array}{c}\text { Code } \\
\text { Stagiaires }\end{array}$ & SM & CJ & LV & ET & OB & KD & PB \\
\hline DNL & $\begin{array}{c}\text { Physique } \\
\text { chimie }\end{array}$ & $\begin{array}{c}\text { Physique } \\
\text { chimie }\end{array}$ & $\begin{array}{c}\text { Physique } \\
\text { chimie }\end{array}$ & $\begin{array}{c}\text { Histoire } \\
\text { géographie }\end{array}$ & $\begin{array}{c}\text { Communication } \\
\text { Action bureau. }\end{array}$ & $\begin{array}{c}\text { Physique } \\
\text { chimie }\end{array}$ & $\begin{array}{c}\text { Génie } \\
\text { civil }\end{array}$ \\
\hline LVE & Anglais & Anglais & Anglais & Espagnol & Italien & Anglais & Anglais \\
\hline
\end{tabular}

Tableau 3 : codification et statut des stagiaires interrogés

A partir de quarante portfolios de compétences, destinés à l'évaluation de l'année de stage, récupérés en fin d'année scolaire 2007/2008 dans le cadre de la thèse (Michaud, 2010), nous comptons sept objets décrits et analysés par les stagiaires qui traitent de la DNL en anglais ou en espagnol, soit $3,5 \%$ des objets rencontrés (7/200). Ces objets sont relatifs à la formation préparant à la certification DNL, à la prise en responsabilité d'une classe DNL en LVE ou à un stage à l'étranger. Les stagiaires ayant fournis dans leur portfolio un objet qui porte sur la DNL en LVE sont définis dans le Tableau 4.

\begin{tabular}{|l|c|c|c|c|c|c|c|}
\hline $\begin{array}{c}\text { Code } \\
\text { Stagiaire }\end{array}$ & SM & CJ & LV & ET & XD & KD & PB \\
\hline DNL & $\begin{array}{c}\text { Physique } \\
\text { chimie }\end{array}$ & $\begin{array}{c}\text { Physique } \\
\text { chimie }\end{array}$ & $\begin{array}{c}\text { Physique } \\
\text { chimie }\end{array}$ & $\begin{array}{c}\text { Histoire } \\
\text { géographie }\end{array}$ & $\begin{array}{c}\text { Physique } \\
\text { chimie }\end{array}$ & $\begin{array}{c}\text { Physique } \\
\text { chimie }\end{array}$ & $\begin{array}{c}\text { Génie } \\
\text { civil }\end{array}$ \\
\hline LVE & Anglais & Anglais & Anglais & Espagnol & Anglais & Anglais & Anglais \\
\hline Niveau & $\begin{array}{c}\text { Classe } \\
\text { Seconde }\end{array}$ & $\begin{array}{c}\text { Classe } \\
\text { Seconde }\end{array}$ & $\begin{array}{c}\text { Classe } \\
\text { Seconde }\end{array}$ & $\begin{array}{c}\text { Classe } \\
\text { Seconde }\end{array}$ & $\begin{array}{c}\text { Pas de } \\
\text { classe dnl }\end{array}$ & $\begin{array}{c}\text { Pas de } \\
\text { classe dnl }\end{array}$ & $\begin{array}{c}\text { Pas de } \\
\text { classe dnl }\end{array}$ \\
\hline $\begin{array}{l}\text { Stage à } \\
\text { l'étranger }\end{array}$ & non & non & non & non & $\begin{array}{c}\text { Stage } \\
\text { aux USA }\end{array}$ & non & non \\
\hline
\end{tabular}

Tableau 4 : codification des stagiaires ayant fournis un texte du portfolio sur la DNL

L'analyse des portfolios et du questionnaire est faite à partir du modèle théorique KnowledgeValue-Practice (Clément, 2006). Les résultats sont présentés selon les trois registres de ce modèle. La pluralité des situations d'enseignement en DNL et le croisement de celles-ci avec les réponses données par les experts doivent nous aider à caractériser la contextualisation didactique aux enseignements de la DNL en LVE.

\section{Résultats et discussion}

Nous discutons dans la première partie les résultats du questionnaire transmis aux formateurs DNL et aux professeurs stagiaires. Dans la seconde partie, nous utilisons les portfolios des professeurs stagiaires pour dégager les obstacles rencontrés et solutions proposées dans cette pratique nouvelle pour eux.

\subsection{Le questionnaire}

\subsubsection{Registre des connaissances : $K$}

\section{Contextes documentaires différents d'une discipline à l'autre}

Les situations sont assez différentes entre les disciplines. D'un côté, on utilise des ouvrages biculturels (franco-allemand) et d'un autre côté, on a recours à des morceaux choisis sur des ouvrages en langue étrangère en fonction des thèmes étudiés. L'utilisation de manuels étrangers de la discipline prend en compte une double contrainte, celle de la langue étrangère 
et celle de la discipline. Par exemple, l'enseignement d'histoire géographie en allemand nécessite de s'appuyer sur des manuels utilisés en Allemagne avec des élèves deux ans plus jeunes (VD). Cela est dû à la part importante de la langue dans l'apprentissage de l'histoire géographie et aussi de la difficulté de cette langue étrangère. L'enseignement des mathématiques en anglais ne pose pas le problème de niveau en langue étrangère car le vocabulaire utilisé est sensiblement le même et le contexte lexical nouveau assez proche du français et de certaines racines latines et germaniques communes aux deux langues (LP).

En fonction des disciplines les supports sont abondamment utilisés dans la langue étrangère : vidéos, cartes, statistiques, journaux, reportages sur des événements internationaux. «En DNL, j'aborde des thèmes scientifiques à travers des vidéos (de quelques minutes), des documents audio ou des articles »(JLJ). Bien souvent, le professeur doit faire preuve d'imagination en s'appuyant sur l'actualité locale, régionale et internationale et l'authenticité des médias utilisés car il n'existe pas de manuel pour l'enseignement de la DNL en LVE.

\section{Effets de contextes : Le renforcement de la compréhension des concepts dans la LE}

Le fait d'aborder un concept scientifique en L2 renforce sa compréhension (Moore, 2001). I1 peut s'agir d'une approche supplémentaire déjà faite en L1 : "Oui, je pense car les élèves en section européenne ont l'occasion d'aborder un concept scientifique une deuxième fois et par une autre approche " (JLJ). La L2 peut être dans certains cas une langue plus imagée que la L1, offrant ainsi un contexte de compréhension supplémentaire (FP). A titre d'exemple une inspectrice faisait remarquer que le terme : «quintessence est assez abscond en français »; le terme équivalent en allemand "der Inbegriff, traduction mot à mot: le sens à l'intérieur, le concept à l'intérieur/de l'intérieur) est plus parlant» (FP). Mais il est important pour l'élève qu'il puisse " associer le concept au mot qui le désigne dans sa langue étrangère, pour qu'il soit bien ancré dans le réseau de connaissance »(PG). Les deux exemples précédents montrent le renforcement de la compréhension de concept dans le contexte de la LE.

\section{Contextualisation didactique : une modification des contenus de la DNL dans la LE}

L'ensemble des enseignants affirme que les contenus et les pratiques de la DNL en LVE sont modifiés du fait de l'enseignement en langue étrangère. Il y a nécessité de problématiser les moyens de médiation pour permettre les apprentissages conjoints de la langue et des concepts disciplinaires :

«La préparation de cours doit inclure l'analyse des moyens sémiotiques indispensables, l'analyse des connaissances langagières des élèves, combiner analyse disciplinaire et linguistique, rajouter (en cas de besoin) des activités favorisant l'appropriation du langage » (RC).

Les contenus diffèrent de ceux qui auraient été enseignés en L1. La difficulté d'apprentissage de la langue et l'importance de la langue écrite et orale pour l'enseignement de la discipline peuvent limiter les contenus à l'essentiel du référentiel :

«En fonction du niveau de langue de la classe, on peut être forcé de se limiter à l'essentiel. La didactique des langues étrangères a élaboré un certain nombre d'outils/de stratégies qu'on peut transférer dans les classes de type DNL » (RC).

La didactique disciplinaire du pays de la langue cible est majoritairement connue des enseignants DNL mais est peu utilisée dans la classe. Un exemple en physique d'une enseignante en stage en Angleterre, qui racontait qu'au lycée le professeur utilisait largement le brainstorming et la manipulation devant le groupe classe alors qu'en France, on fait 
manipuler les élèves en autonomie en physique et chimie (CJ). Dans ce cas, l'approche comparative de la didactique disciplinaire entre la France et l'Angleterre permet une prise de recul sur les pratiques professionnelles de l'enseignant. Enfin, d'autres s'appuient sur les différences de la culture professionnelle du pays pour modifier les contenus d'enseignement: "Oui, les contenus diffèreront: par exemple nous pourrons étudier les différences entre la lettre commerciale en France et en Italie, au niveau du fond et de la forme " (OB). Pour conclure ce paragraphe, si les contenus d'enseignement de la DNL dans la LVE sont transformés par rapport à un enseignement en L1, ils doivent cependant respecter le référentiel de la DNL tout en apportant une dimension culturelle.

\subsubsection{Registre des pratiques : $P$}

\section{Alternance codique sur une même séance : fonction didactique ou adidactique}

Il y a autant de pratique de la DNL qu'il y a de disciplines, de situations variées et de collègues qui l'enseignent. Certains pratiquent la totalité de la discipline dans la LE, d'autres réservent des horaires exclusifs de la discipline en L1 ou LE.

L'alternance de la langue est d'une manière générale peu pratiquée dans un même cours. Cette alternance est une fonction de régulation de l'enseignement ou de stratégie d'appui de la DNL : passage de consignes, incompréhension des élèves ou suggestion d'un point d'arrêt. Elle est appelée dans ce cas fonction adidactique par certains auteurs confrontés au problème de l'alternance codique (Anciaux, 2010).

Les registres de cette alternance se situent sur des points précis tels que l'impérieuse nécessité de la compréhension de règles de sécurité en physique par exemple: "Pour un TP, notamment au niveau des consignes de sécurité, je pense qu'il est essentiel de commencer par traduire systématiquement ce qu'on attend »(CJ).

En sciences et techniques, une collègue peut utiliser le français pour expliquer une règle de grammaire ou la traduction d'un terme technique que les élèves ne connaissent pas encore.

"Quand quelques traductions de mots techniques sont nécessaires ou pour faire un point de grammaire (remise à niveau à destination des plus faibles) 》(BS). L'utilisation de la L1 peut se faire en dernier recours « après avoir épuisé les autres méthodes comme les synonymes en L2, une définition en L2, une image ou illustration préalablement prévue dans un Powerpoint, etc. » (BS).

L'alternance codique peut être utilisée pour aider à la mémorisation des concepts dans une fonction didactique (Moore, 1996) et/ou dans une stratégie de contraste qui identifie les points communs ou les différences entre la L1 et la LE (Causa, 1996) :

"Lorsque je souhaite faire un parallèle sur une même situation entre anglais et français, cela peut être pertinent (même si j'y ai faiblement recours). En mathématiques, il est par exemple important de remarquer qu'une grande partie des termes mathématiques se retrouvent à l'identique en anglais on peut ainsi se concentrer sur les faux amis et les différences et pour le reste angliciser le terme français (du moins à l'oral) » (LP). 
A travers les exemples précédents, l'alternance codique permet le passage d'un contexte de langue à un autre tout en produisant des effets sur les contextes et le contrat didactique. On peut ainsi parler de didactisation de l'AC dans la discipline linguistique et non linguistique.

\section{Effet de l'enseignement de la DNL dans la LE : vers une différenciation pédagogique accrue}

La compréhension de la DNL dans la LE requiert de la part de l'enseignant une attention toute particulière qui s'appuie sur les feedbacks produits par la classe. Cela demande aux enseignants DNL de pratiquer une différenciation pédagogique où «la pédagogie est constamment confrontée à la question de l'intercompréhension. On peut donc supposer que l'enseignant s'interroge et s'adapte davantage aux besoins des apprenants » (RC). La progression didactique s'en trouve ralentie. L'approche en langue étrangère de la discipline "nécessite de la part de l'enseignant d'intégrer une compétence langagière souvent négligée en classe monolingue » (RC). Il y a multiplication des canaux sémiotiques pour accéder à la signification. La double entrée sémiotique dans chacune des langues permet une nouvelle entrée qui accentue voire précise le concept.

\subsubsection{Registre des valeurs : $V$}

\section{Effets de contextes : ouverture scientifique et renforcement du sens dans la discipline}

La pédagogie DNL est une pédagogie d'ouverture au monde scientifique et sur le monde :

«Autre exemple: le fonctionnement d'une centrale nucléaire ne figure pas au programme de SPC en TS. Mes collègues et moi traitons ce thème superficiellement voire pas du tout. En DNL on peut prendre le temps d'étudier en profondeur le fonctionnement d'une centrale nucléaire et de clore le thème par un débat sur l'énergie nucléaire (en anglais évidemment !) » (JLJ).

Les enseignants vont jusqu'à utiliser des situations problèmes en mathématiques plus inattendues "dans lesquelles les mathématiques sont mises en valeur, soit en tant qu'outil de résolution ou de modélisation (pourquoi les abeilles utilisent des alvéoles hexagonales?" (LP). La DNL conduit à un renforcement du sens donné aux apprentissages par la décontextualisation des objets d'enseignement qui mettent en valeur la DNL.

\section{Effets de contextes : ouvertures culturelles et réflexives}

La perspective culturelle est présente :

"Perspective culturelle différente: en histoire géographie par exemple, les documents supports utilisés étant en langue allemande, ils permettent de voir un même événement sous un autre angle. En mathématiques, les approches, la manière de présenter les choses sont différentes d'un pays à l'autre, ce qui peut aider les élèves à comprendre. En tout cas, il s'agit de changer de point de vue, ce qui est très formateur » (FP).

Le changement de point de vue par une approche biculturelle par le contexte de la L1 et de la LE peut conduire au développement de la réflexivité.

En génie civil par exemple, cette entrée culturelle peut quelquefois démotiver certains élèves qui accrochent davantage la proximité locale : 
" J'ai remarqué que certains étudiants accrochaient plus au cours si on basait le cours sur des cas d'étude français, voir locaux! Ce choix doit être adapté en fonction de la curiosité intellectuelle du public étudiant, et peut avoir une influence non négligeable sur la motivation des élèves et l'acquisition du cours » (BS).

Cela s'explique par le fait que le contexte local renvoie à des techniques habituelles de construction qui ne déstabilisent pas les élèves. Ces techniques peuvent différer dans un pays étranger où est parlée la LE (exemple: construction en bois dans les pays nordiques, construction en briques au Royaume Uni, construction en maçonnerie en France, etc.). Le contexte local lié à la L1 et/ ou éloigné de la LE peut être choisi en fonction des objectifs d'apprentissage et des préoccupations des élèves.

\subsection{Les portfolios}

\subsubsection{Registre des pratiques : $P$}

\section{Obstacles rencontrés par les stagiaires et proposition de prise en compte des effets de contextes pour améliorer l'apprentissage des élèves}

\section{Difficultés rencontrés par les stagiaires}

L'analyse des réflexions des stagiaires dans les portfolios met en évidence leurs difficultés sur la maîtrise de la langue cible. En effet, si la maîtrise de la didactique disciplinaire semble acquise, la langue seconde représente un double obstacle au niveau de sa maitrise et au niveau de son enseignement. Sur ce dernier point, ils font état des réflexions suivantes :

- Progresser dans l'étude du vocabulaire de la discipline en adéquation avec la progression disciplinaire ;

- Faire passer les nuances de la LE : simplicité, clarté et efficacité ;

- Eviter les traductions de la LE vers la L1;

- Pratiquer une évaluation de la discipline dans la LE plus globale en ne focalisant pas sur le mot à mot dans le discours. "La première tentation est d'essayer d'évaluer sur la pratique générale de la langue. Je me suis vite rendu compte que ma formation ne me permettait pas de réaliser ce type d'évaluation" (LV). Un stagiaire évalue par exemple les mots nouveaux de la discipline dans une interrogation écrite en anglais par le remplissage d'une grille de mots croisés.

\section{Recherche de collaboration avec le professeur de langue}

Cela conduit les stagiaires à chercher des réponses auprès du collègue qui enseigne la langue étrangère dans l'équipe pédagogique: "Une collaboration étroite avec le professeur d'anglais est une étape essentielle » (SM). Causa (2009 : 186) parle de travail en tandem dans une pédagogie de projet avec le professeur de langue. L'apprentissage ludique est favorisé en classe DNL pour motiver les élèves et "délègue souvent au professeur de langue les apprentissages plus rébarbatifs de la langue, grammaire, forme syntaxique... »(LV).

\section{Importance de la communication orale}

$\mathrm{Au}$ niveau de la communication, ils font ressortir l'importance de la communication orale dans l'enseignement de la DNL. Cette communication permet de vérifier la compréhension des élèves : "Pour pouvoir s'adapter aux élèves, il faut animer la classe de façon à avoir un 
retour pour vérifier si les termes utilisées sont compris" (SM). La communication est intégrée aux besoins disciplinaires :

"En sciences physiques, la possibilité de s'exprimer est offerte par la description d'une expérience, du matériel utilisé, du protocole suivi, ou encore par la proposition d'hypothèses, l'exposition de ces observations, de ses conclusions tout en les argumentant. Pour l'expression écrite, des productions écrites courtes peuvent être demandées et corrigées suite à des expérimentations. » (SM).

Dans cet exemple, le contexte disciplinaire en physique conduit à développer dans la LE un raisonnement scientifique qui s'appuie sur les compétences linguistiques et pragmatique de la langue.

\subsubsection{Registre des connaissances : $K$}

\section{Le renforcement de la compréhension des concepts dans le contexte de la L2}

Au niveau des connaissances, il existe des situations où par exemple l'usage de la langue seconde rend plus facile la compréhension du concept d'élément chimique en anglais :

"J'ai choisi d'enseigner cette leçon particulière en anglais car nombre de mes élèves avaient des difficultés pour mémoriser les symboles des éléments. Par exemple, ils symbolisaient souvent le sodium par So. Grace à ce TP, ils comprirent pourquoi l'azote est symbolisé par $N$ et non par A (le mot azote se traduit par nitrogen' en anglais). » (CJ)

\section{Et aussi le renforcement de la réflexion dans le contexte de la $\mathbf{L 1}$}

Sur le plan métalinguistique, la réflexion sur la compréhension de la langue étrangère amène certains stagiaires à étendre leur réflexion sur la langue première "La langue seconde est un obstacle, et j'ai réfléchi que la langue première peut être aussi un obstacle dont l'enseignant n'a pas forcément conscience » (CJ). Sur le même plan métalinguistique, une stagiaire fait une comparaison entre les apprentissages de la langue à long terme et ceux de sa discipline supposés à priori à court terme :

«En sciences physiques, je raisonnais plus sur des objectifs à court terme, un objectif sur une leçon par exemple. Ma formation DNL et la réflexion que j'ai pu avoir sur le développement des compétences linguistiques à long terme m'a permis d'élargir ma vision dans ma propre discipline » (SM).

\subsubsection{Registre des valeurs : V}

Le registre des valeurs n'est pas abordé par les stagiaires dans les portfolios et le questionnaire. Il semble bien que les difficultés rencontrées dans le registre de la pratique soient trop prégnantes pour permettre une prise de conscience des valeurs inter et socioculturels dans l'enseignement de la DNL.

\section{Conclusion}

Nous regroupons les résultats précédents des questionnaires et des portfolios dans les trois champs du modèle KVP. Nous dégageons les généralités d'une pratique d'enseignement en contexte de la DNL dans la LVE (cf. Figure 2). 


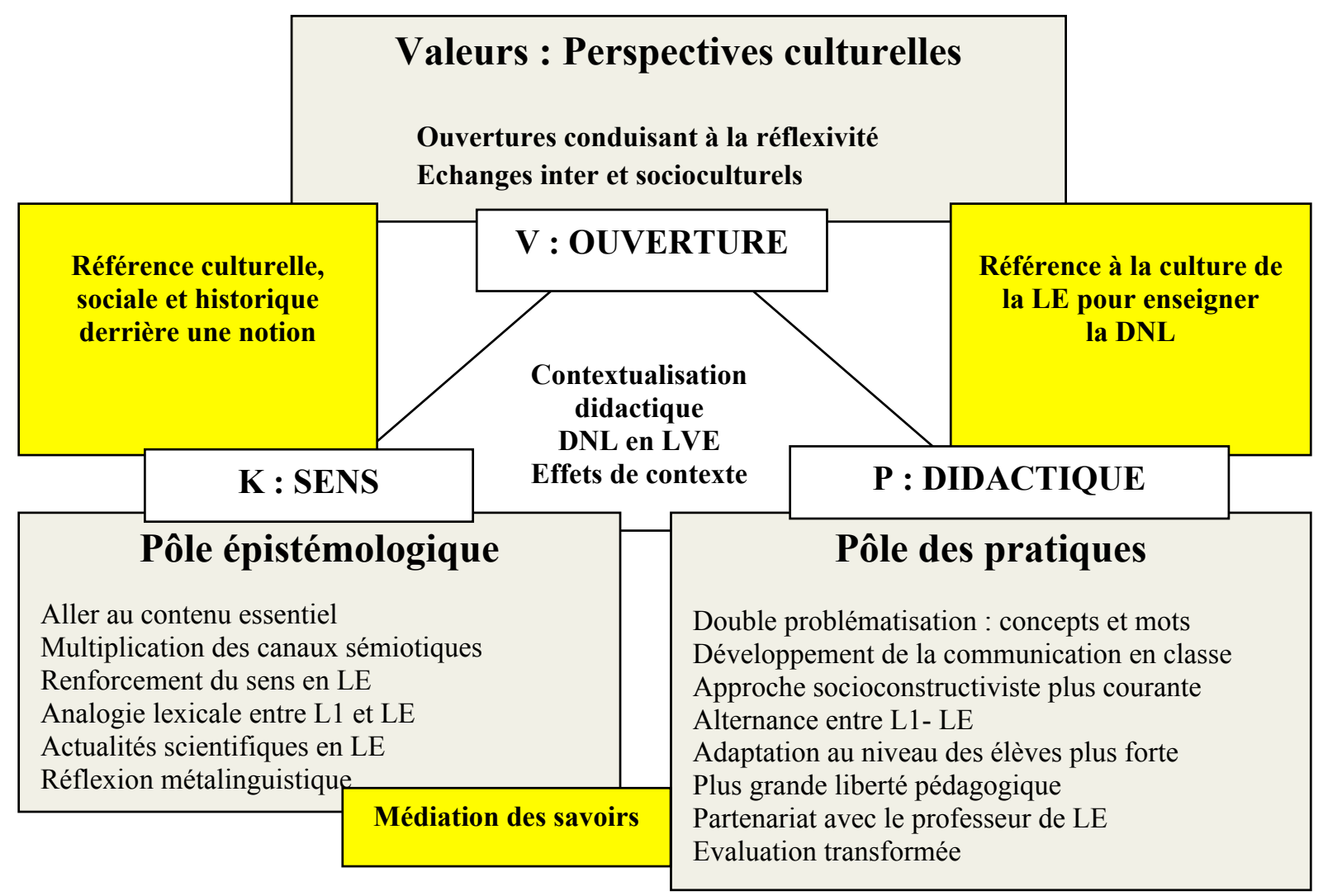

Figure 2 : Présentation des résultats dans le modèle KVP

Concernant le registre des pratiques $(\mathrm{P})$, le métier de l'enseignant est largement convoqué et conduit à une double réflexion sur le sens de la forme linguistique d'une part et sur l'adéquation de cette forme avec le contenu en faisant référence à la bi-focalisation de Bange (1992) d'autre part. Cela a pour conséquence une double problématisation des processus de médiation au niveau de la pratique enseignante et la multiplication de ces outils de médiation pour une meilleure adaptation au niveau des élèves. L'enseignement est alors adapté au contexte dans lequel il se déroule, il est possible de parler d'effets de contextes. Nous avons relevé d'autres effets de contextes dans le champ des pratiques : l'alternance codique entre L1 et LE avec les fonctions didactiques et communicatives (Moore, 1996), le partenariat avec le professeur de LE et une évaluation transformée de la DNL dans LVE par rapport à celle de la DNL dans la L1 qui pourrait être une piste à approfondir dans le cadre de recherches futures.

Concernant les contenus d'enseignement $(\mathrm{K})$, ils sont largement transformés du fait de l'enseignement de la DNL en LVE. Ils sont à didactiser en respectant le référentiel de la DNL (Duverger, 2009) tout en apportant une dimension culturelle qui associe des documents authentiques et récents en lien avec des objets de la langue étrangère.

Le registre des valeurs (V) conduit à une réflexion accrue par décentration du contexte culturel en comparant des événements, des processus techniques attenant aux pays de la L1 et de la LE et ou en portant le double regard culturel de la L1 et de la LE sur le monde.

Cette contextualisation didactique des DNL en LVE, dont nous avons essayé de dégager les effets de contextes et les contours communs, serait à étudier discipline par discipline. Les DNL ont elles-mêmes des didactiques spécifiques qui leur servent de colonne vertébrale et qui 
se voient transformer différemment du fait de leur enseignement dans une langue étrangère. Cet enseignement est dans les usages davantage explicité à l'oral en LE qu'il le serait en L1. Son apprentissage s'en trouve de fait transformé et demande de la part de l'enseignant une réflexion approfondie au niveau de la langue LE pour laquelle il n'a pas été formé. Ce qui pose une question centrale celle de la formation des enseignants (Causa, 2009 : 185).

Ces questions restent d'actualité dans la réforme des lycées dans les voies technologiques qui prônent une heure par semaine d'enseignement technologique en langue étrangère en classe de première et terminale. On peut penser que dans un avenir plus ou moins proche une généralisation de la DNL en LVE devienne le moyen d'apprentissage des langues étrangères avec un objectif linguistique aussi important que disciplinaire. En quoi cela pourrait-il à terme modifier l'enseignement des langues étrangères?

\section{Références bibliographiques}

Anciaux, F. (2010). Vers une didactique de l'alternance codique aux Antilles françaises. Manuscrit publié dans les actes du Colloque international «Spécificités et diversité des interactions didactiques : disciplines, finalités, contextes » Université Lyon 2 -ICARINRP- CNRS.

Bange, P. (1992). À propos de la communication et de l'apprentissage en L2, notamment dans ses formes institutionnelles. Acquisition et interaction en langue étrangère, Aile, 1, 5385.

Causa, M. (2009). Enseignement d'une LE et d'une DNL : mettre en place une " compétence discursive ». Synergies Roumanie, 4, Gerflint.

Causa, M. (2007). L'indispensable alternance codique des langues. Le français dans le monde, 351, 18-19.

Causa, M. (2002). L'alternance codique dans l'enseignement d'une langue étrangère: Stratégies d'enseignement bilingues et transmission de savoir en langue étrangère. Bern : Peter Lang.

Causa, M. (1996). L'alternance codique dans le discours de l'enseignant. Entre transmission de connaissance et interaction. Les cahiers du Cédiscor, 4, 111-129.

Clément, P. (2006). Didactic Transposition and KVP Model : Conceptions as Interactions Between Scientific knowledge, Values and Social Practices. ESERA Summer School, IEC, Université Minho, Braga (Portugal), 9-18.

Coste, D. (2003). Construire des savoirs en plusieurs langues. Les enjeux disciplinaires de l'enseignement bilingue. Site consulté le 26 mai 2012 : http://www.adeb.asso.fr/archives/problematique/dnl/Coste_Santiago_oct03.pdf.

Duverger, J. (2009). Favoriser l'alternance des langues. Le français dans le monde, 362, 2628.

Duverger, J. (2007). Didactiser l'alternance des langues en cours de DNL. Tréma, 28, 81-88.

Duverger, J. (2005). L'enseignement en classe bilingue. Paris : Hachette.

Duverger, J. (2004). Lire, Ecrire, Apprendre en deux langues. Les Actes de Lecture, 85.

Eyssautier-Bavay, C. (2004). Le portfolio en éducation : concept et usages. Grenoble : PDF, 13.

Forquin, J-C. (1989). Ecole et culture. Le point de vue des sociologues britanniques. Bruxelles : De Boeck.

Giordan, A. (2005). Vive la motivation ? Cahiers pédagogiques, 431, 58.

Goupil, G. (1998). Portfolios et dossiers d'apprentissages. Montréal, Chenelière : Mc GrawHill. 
Michaud, C. (2010). Le Portfolio: un en(je)-u de formation et de développement professionnel. Thèse de Doctorat non publiée. Lyon : Université Claude Bernard Lyon 1.

Michaud, C. et Alin C. (2010). Le portfolio: analyse métaphorique et identité professionnelle. Dans G. Baillat, D. Niclot et D. Ulma (dir.), La formation des enseignants en Europe : Approche comparative (p. 173-192). Collection Pédagogie en développement. Bruxelles : De Boeck Université.

Michaud, C. et Alin C. (2008). E-portfolio et Construction de l'identité professionnelle des enseignants stagiaires. Communication présentée à la $2^{\text {ème }}$ Conférence Pan-Américaine et Francophone - Université Concordia, Montréal, Canada.

Moore, D. (2001). Une didactique de l'alternance pour mieux apprendre? Etudes de linguistique appliquée, 121(1), 71-78.

Moore, D. (1996). Bouées transcodiques en situation immersive, ou comment interagir avec deux langues quand on apprend une langue étrangère à l'école. Acquisition et interaction en langue étrangère, Aile, 7, 95-121.

Piaget, J. (1970). Psychologie et épistémologie. Paris : Gonthier Denoël.

Rabardel, P. (2005). Instrument subjectif et développement du pouvoir d'agir. Dans P. Rabardel et P. Pastré (dir.), Modèles du sujet pour la conception. Dialectiques activités développement (p. 11-29). Toulouse : Octarès.

Vygotski, L.S. (1934). Pensée et langage. Paris : Éditions Sociales.

Winsor, J.T., et Ellefson, B.A. (1995). Professionnal portfolios in teacher education: An exploration of their value and potential. The Teacher Educator, 31, 68-74.

Wolf, K.F. (1991). Teaching Portfolios : Synthesis of Research and Annotated Bibliography. San Francisco (CA) : Far west lab. For Educational and Development. Document ERIC ED 343890. 


\section{Annexe 1 : Enquête DNL - Formations IUFM années 2007-2010}

Année de réussite de la certification : (à compléter)

Discipline de la certification DNL : $\quad$ (à compléter)

Langue étrangère :

(à compléter)

- Avez-vous eu une classe en responsabilité en DNL pendant ou depuis l'année de la certification ? Oui - Non.

- Si oui, en quelle(s) année (s) ?: ～(à compléter)

\section{1- La pratique de la langue}

- Y a-t- il des séances complètes en français et d'autres en langue étrangère pour enseigner la DNL ? Oui - Non.

- Pratiquez-vous une alternance de la langue dans une même séance et selon quelles modalités?

- En quoi l'alternance de la langue française-langue étrangère sur une même séance facilite les apprentissages des élèves? Quels sont les moments choisis pour l'une ou l'autre des situations?

- Quels sont selon vous les bénéfices de l'enseignement DNL par rapport à un enseignement classique de la discipline en français?

\section{2- Les manuels et documents pédagogiques}

- Utilisez-vous des manuels, articles de revues, vidéos de la langue étrangère pour enseigner votre discipline ? Oui - Non. Lesquels?

- Si oui : Est-ce que les stratégies d'enseignement de la discipline diffèrent entre le manuel français et celui de la langue étrangère?

- Est-ce que cela change les contenus et / ou vos pratiques d'enseignement ? Oui - Non. En quoi ?

\section{3- Les contenus disciplinaires}

- Est-ce que les contenus disciplinaires dans le cadre de la DNL se trouvent réduits à ceux que vous auriez enseignés en français ? Oui - Non. Est-il possible d'ouvrir sur les contenus disciplinaires utilisés dans le pays de la langue étrangère ? Si oui comment?

- Est-ce que les entrées disciplinaires peuvent se faire à partir de la culture du pays étranger? Oui - Non. Sous quelles formes?

- Est-ce que le fait de comprendre un concept scientifique dans la langue étrangère renforce et approfondit sa compréhension dans la langue de scolarisation initiale ?

- Donnez-vous les traductions des contenus disciplinaires acquis dans la langue étrangère?

- Y a-t-il une didactique spécifique pour l'enseignement des disciplines non linguistiques ? Oui - Non

- Comment peut-on la caractériser dans vote discipline?

4- Le développement professionnel (question posée uniquement aux stagiaires)

- Dire en quoi l'enseignement de la DNL vous a permis de comprendre certaines choses, de réfléchir dans votre discipline, de voir votre métier sous un angle différent y compris pour les autres disciplines 